\title{
Computational modeling of heat and mass transfer processes in combustion chamber at power plant of Kazakhstan
}

\author{
Reinhard Leithner ${ }^{1}$, Aliya Askarova ${ }^{2}$, Saltanat Bolegenova ${ }^{2}$, Symbat Bolegenova $^{2}$, Valeriy Maximov $^{2 a}$, Shinar Ospanova $^{3}$, \\ Aigul Ergalieva ${ }^{3}$, Aizhan Nugymanova ${ }^{3}$, Meruyert Beketayeva ${ }^{3}$ \\ ${ }^{1}$ Technical University of Braunschweig, Institute of Energy and Process Systems Engineering, D-38023 Braunschweig, Germany \\ ${ }^{2}$ SRI of Experimental and Theoretical Physic, Al-Farabi Kazakh National University, 050040, Almaty, Kazakhstan \\ ${ }^{3}$ Al-Farabi Kazakh National University, Department of Physic and Technology, 050040 Almaty, Kazakhstan
}

\begin{abstract}
In this paper the results obtained by the method of numerical modelling of Ekibastuz coal burning in furnace of Kazakhstan Power Plant. Numerical experiment was carried out on the basis of three-dimensional equations of convective heat and mass transfer, taking into account the heat propagation, heat radiation, chemical reactions and multiphase structure of the medium to predict the influence of different water content in coal on overall furnace operation and formation of combustion products.
\end{abstract}

\section{Introduction}

The power system of Kazakhstan is presented mostly by thermal power stations more than $70 \%$ of total amount of the electric power are generated. Thermal power stations work on solid fuel when pulverized coal combustion is predominant. Also natural gas and black oil can be used. Tendency of low grade coals usage to increase in domestic industry, it becomes especially important to develop and introduce new energy-saving technologies of solid fuel consumption and reduction in pollutant substance emissions. Applied technology of direct burning of low-grade coals in furnaces does not provide demanded reliability of working equipment and protection of environment from harmful combustion products due to approximately $50 \%$ of pollutant emissions from stationary sources comes from thermal power enterprises and about $33 \%$ are emitted by ferrous and non-ferrous metallurgy and mining.

Industrial implementation of any new technology is not possible without preliminary analysis of advantages and disadvantages suggested method. The rapid development in computer sciences gives the advance to computational techniques to be used for simulation of complex combustion processes in industrial furnaces.

Products of combustion contain different harmful substances and the emission of these components grows in to a great problem. Industrial development causes an increase in carbonaceous fuels' consumption. These fuels contain harmful and poisonous components such as carbonic oxide (CO), nitric oxide (NO), sulphur dioxide $\left(\mathrm{SO}_{2}\right)$, lead combinations and different hydrocarbons etc.
To decrease emissions of harmful substances various methods are applied, including special fire regimes (organization of combustion process), which suppresses the formation of harmful substances in flame and twostage burning, when the burners work with low air surplus. In this way numerical experiments became one of the most effective and suitable means for detail analysis and in-depth study of physical and chemical phenomena.

In contrast to construction of an operating reduced model of the chamber, the three-dimensional modelling with the application of modern computer technology enables to carry out deep analysis of all chamber's parameters and save time and finances. At the same time, without additional inputs, it is possible to obtain the full set of characteristics of a convective heat and mass transfer process in reactive media, intervene flexibly in the process at any stage and reproduce separate technical solutions (the configuration of the fire chamber and assembly and construction burners), to model the formation of harmful dust and gaseous emissions and to investigate the influence of previous preparation of coal on its ignition and combustion stabilization.

\section{Mathematical models}

Combustion of coal-dust flame is a very complex physical-chemical process for mathematical analysis. In the boilers, where coal-dust fuel burns down in the air stream, many interrelated process occur. They are: complex aerodynamics, combustion under conditions of constantly changing temperature and component

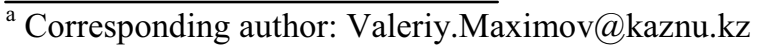


concentrations, heat exchange between the flame and the chamber surface. Simultaneously formation of carbon, nitrogen and sulphur oxide, corrosion hazardous and carcinogenic substances occurs.

A numerical experiment was carried out on the basis of three-dimensional equations of convective heat and mass transfer, taking into account the heat propagation, heat radiation, chemical reactions and the multiphase structure of the medium. To describe 3-D motion of reacting flows in chambers, the following set of differential equations was used [1]:

Continuity equation:

$$
\frac{\partial \rho}{\partial \tau}=-\frac{\partial}{\partial x_{i}}\left(\rho u_{i}\right)
$$

Momentum conservation law:

$$
\frac{\partial}{\partial \tau}\left(\rho u_{i}\right)=-\frac{\partial}{\partial x_{j}}\left(\rho u_{i} u_{j}\right)+\frac{\partial}{\partial x_{j}}\left(\tau_{i, j}\right)-\frac{\partial p}{\partial x_{j}}+\rho f_{i},
$$

here: $f_{i}$ - volume forces; $\tau_{i, j}-$ strain tensor.

Energy equation:

$$
\begin{aligned}
& \frac{\partial}{\partial \tau}(\rho h)=-\frac{\partial}{\partial x_{i}}\left(\rho u_{i} h\right)-\frac{\partial q_{i}^{r e s}}{\partial x_{j}}+\frac{\partial p}{\partial \tau}+ \\
& +u_{i} \frac{\partial p}{\partial x_{i}}+\tau_{i j} \frac{\partial u_{j}}{\partial x_{i}}+s_{q},
\end{aligned}
$$

here: $h$ - enthalpy; $S_{q}$ - energy source.

Equation for substance components:

$$
\frac{\partial}{\partial t}\left(\rho c_{\beta}\right)=-\frac{\partial}{\partial x_{i}}\left(\rho c_{\beta} u_{i}\right)-\frac{\partial j_{i}}{\partial x_{i}}+R_{\beta},
$$

where $R_{\beta}$ - source of matter.

A standard $k-\varepsilon$ model of turbulence has been used to close off the system and model turbulent viscosity [2,3]:

$$
\begin{aligned}
& \frac{\partial}{\partial \tau}(\bar{\rho} k)=-\frac{\partial}{\partial x_{i}}\left(\overline{\rho u_{i}} k\right)+\frac{\partial}{\partial x_{i}}\left[\frac{\mu_{e f f}}{\sigma_{k}} \frac{\partial k}{\partial x_{i}}\right]+P-\bar{\rho} \varepsilon \\
& \frac{\partial}{\partial \tau}(\bar{\rho} \varepsilon)=-\frac{\partial}{\partial x_{i}}\left(\overline{\rho u_{i}} \varepsilon\right)+\frac{\partial}{\partial x_{i}}\left[\frac{\mu_{e f f}}{\sigma_{\varepsilon}} \frac{\partial \varepsilon}{\partial x_{i}}\right]+ \\
& +c_{\varepsilon 1} \frac{\varepsilon}{k} P-c_{\varepsilon 2} \bar{\rho} \frac{\varepsilon^{2}}{k}
\end{aligned}
$$

Point concentration of solid matter was determined by means of balance equations for monodispersion solid matter with the average particle diameter. To determine the mixture concentration the homogeneous model was used. Velocities of solid particles were considered to be equaled local gas velocity. Numerical model comprises sub-models for turbulent two-phase mixing, gas-phase chemical reactions, heterogeneous coal particle reactions (devolatilization, char oxidation), convective and sixflux radiant [4] heat transfer.

Calculation of turbulent flows with chemical reactions is based on the knowledge of chemical kinetics and modeling methods of turbulent transfer processes. To determine the source term in equation for components which is related to chemical reaction rate it's necessary to model correctly kinetics of chemical reactions. These reactions take place between fuel and oxidant in combustion space of the boiler. Local distribution of reacting components and temperature significantly influence on the reaction rates. The amount of energy, emitted in chemical reactions, the flame temperature and the nature of combustion products are very important characteristics in estimation of solid substances influence. Using the solid particles as fuel is most probable.

\section{Coal combustion}

The coal combustion model is divided into five submodels for drying, pyrolysis (release of volatiles), and combustion of volatiles, carbon monoxide and residual char. Coal devolatilization is considered to run in a single step reaction forming a general hydrocarbon $\mathrm{C}_{\mathrm{x}} \mathrm{H}_{\mathrm{y}}$. The amount and composition of the hydrocarbon species have been taken from elementary and ultimate analysis of the coal. The model of coal dust combustion which is being used in this paper takes into account an integral oxidizing reaction of fuel components down to stable final products of reaction. Intermediate reactions and the formation of intermediate products haven't been taken into consideration. The model of integral reaction was used based on according to the fact that many chemical reactions proceed several stages. The slowest reaction determines the rate of the whole reaction.

The combustion of volatiles in the gas phase is assumed to be controlled by turbulent mixing, using the Eddy Dissipation Concept. The Eddy Dissipation Model according to Magnusson et al. is used to predict the combustion of the volatiles and the carbon monoxide formed during char combustion. Gaseous fuels are treated like volatiles. The char reaction rate is governed by the rate of oxygen diffusion to the surface and the kinetic rate of chemical reaction at the surface.

Simplified models are being used in this paper. These models take into account only chemical reactions of key components since the detail modelling of all passing reactions is possible only in the simple cases, because of large computational costs. Information about reactions and their kinetic data have been taken from [4].

Within the combustion of fossil fuel, nitric oxide is produced by different reaction paths. The main reactions are the oxidation of the molecular nitrogen in the air (thermal NO) and the oxidation of the fuel bounded nitrogen (fuel-NO). To describe the rates of forming and reducing nitric oxide during combustion global reaction schemes proposed by Mitchell and Tarbel [5] are used.

In this paper software package FLOREAN [1, 6-9] for 3 -D modelling of coal-dust combustion in furnaces of real-sized boilers was used. This program enables to calculate velocity components $\mathrm{u}, \mathrm{v}, \mathrm{w}$, temperature $\mathrm{T}$, pressure $\mathrm{P}$, concentration of combustion products and other characteristics of combustion process all over the combustion space and at its exit. Pressure is determined through the connection between the continuity equation 
and the equation of motion by means of Patankar's Simple-method [10].

\section{Results of CFD Studies}

In present work CFD simulation tool was used to simulate and analyse low-grade coal combustion and flow properties of industrial furnaces of Kazakhstan Power Plants. The simulations of the furnaces of steam generators placed at Almaty Power Plant have been carried to predict the influence of different water content in coal on overall furnace operation and formation of combustion products.

Fig. 1 shows temperature distribution along the chamber height for burning of coal with different water content in initial air-fuel stream. The experimental data [11] are given here for coal burning with $7 \%$ moisture. Curve minimums caused by low temperature of fuel mixture supplied in the chamber space through the burners. Differences of calculated and experimental values of temperature are seen in the region of ignition and extinction. Figure 2 shows difference in temperature fields for different initial water content in burning coal. We can see that higher water content causes decrease in temperature in this section of combustion chamber.

Figures 3-8 show picture of fuel burning with the fields of oxygen, carbon oxide (CO) and carbon dioxide $\left(\mathrm{CO}_{2}\right)$, and nitrogen oxide (NO) concentration. The main formation regions of gases in the coal dust torch take place at the level of burners in the central part of the furnace volume.

The maximum difference of calculation and experiment (fig. 7) is seen in the region of ignition and extinction of flying matters. The main reason for this is that there is a determination of the gas burning rate by one stage model of pyrolysis where the set of kinetic constants for all the entire temperature range are used. The figures show that the regions with maximum difference coincide with regions having a high concentration of carbon oxide.

At the chamber furnace outlet we can see that decrease in coal moisture results in $\mathrm{CO}_{2}$ decrease, (fig. 7) and simultaneous $\mathrm{CO}$ increase (fig. 5-6). Oxygen concentration (fig. 3,4) in the total gas mixture at the outlet consequently increase with water content in coal (fig. 3-5). Same development is seen in the whole furnace volume (fig. 3, 5). Difference in $\mathrm{NO}$ formation for studied coal content is seen mostly in the region of burner location. At the furnace outlet the difference in NO concentrations is slight appearing (fig.8). The distribution character of all concentrations, listed in this paper is modelled sufficiently and it agrees with the experimental data [11].

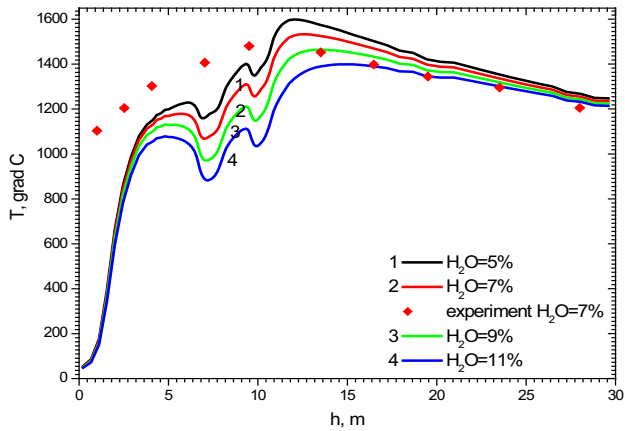

Figure 1. Temperature distribution along the chamber height for coal burning with different water content.

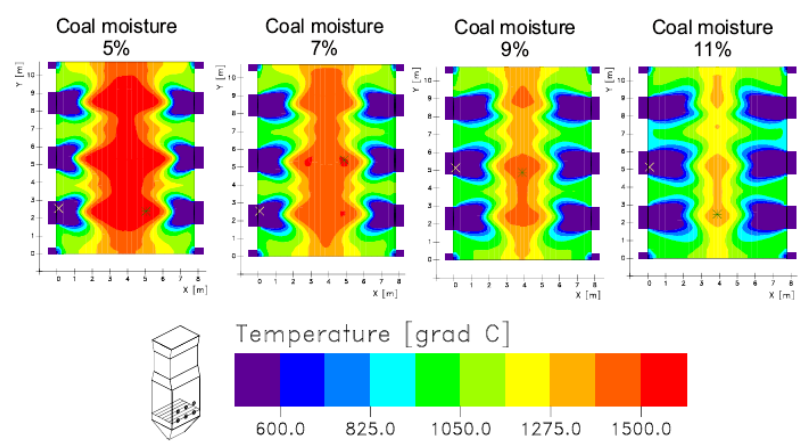

Figure 2. Temperature distribution in cross section of the furnace in the lower level of the burners for coal burning with different water content.

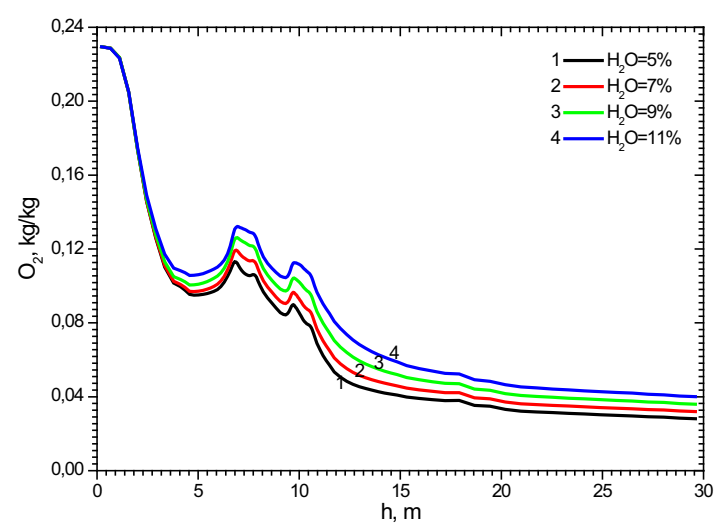

Figure 3. $\mathrm{O}_{2}$ concentration distribution along the chamber height for coal burning with different water content.

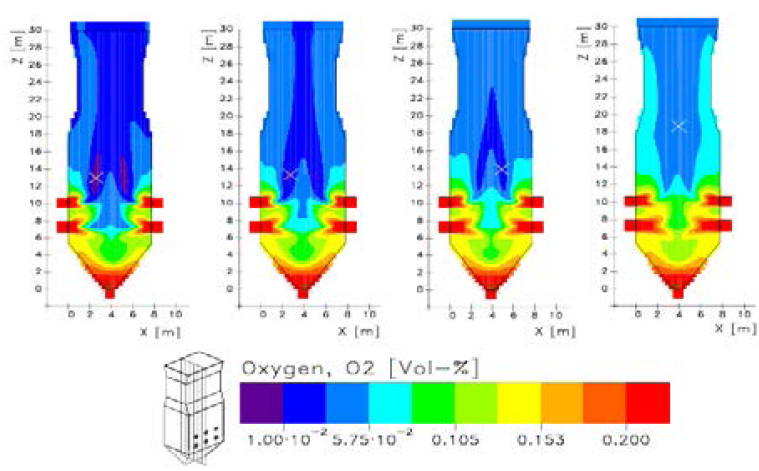

Figure 4. Oxygen concentration distribution in the central section of the furnace for coal burning with different water content. 


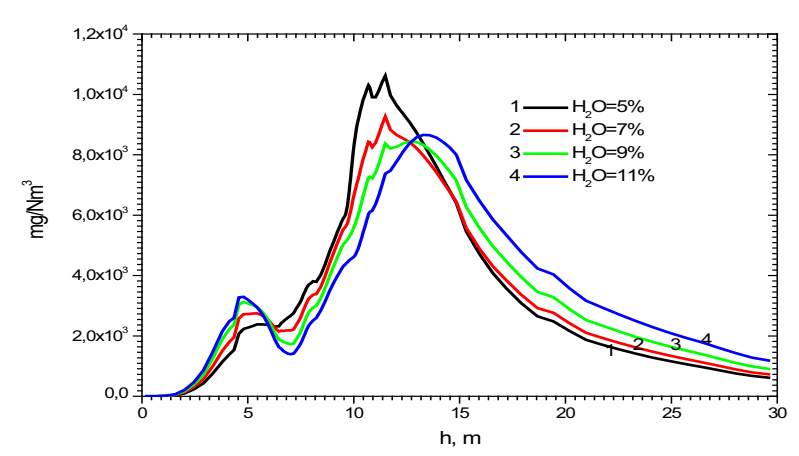

Figure 5. CO concentration distribution along the chamber height for coal burning with different water content.

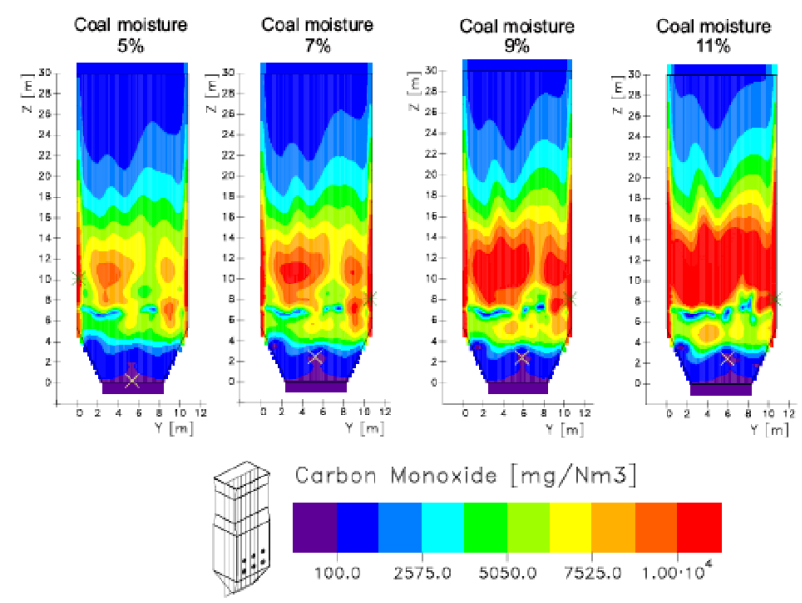

Figure 6. CO distribution in the central section of the furnace for coal burning with different water content.

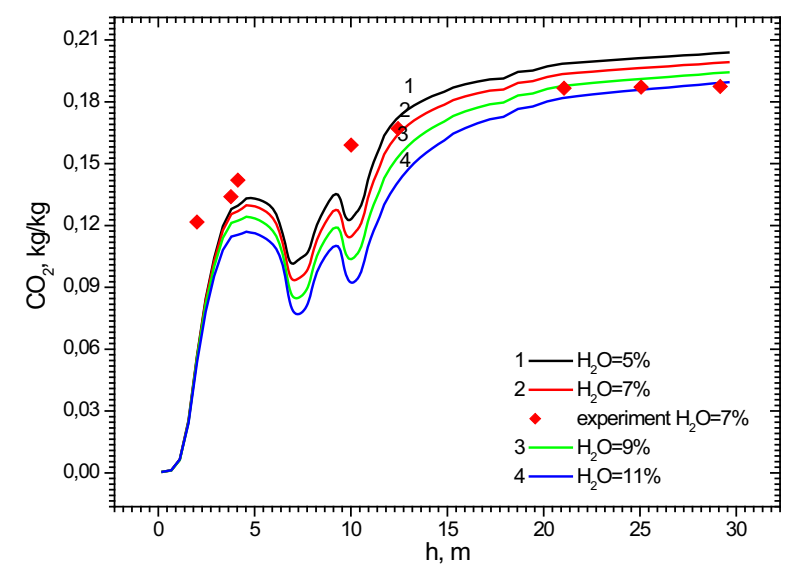

Figure 7. $\mathrm{CO}_{2}$ concentration distribution along the chamber height for coal burning with different water content.

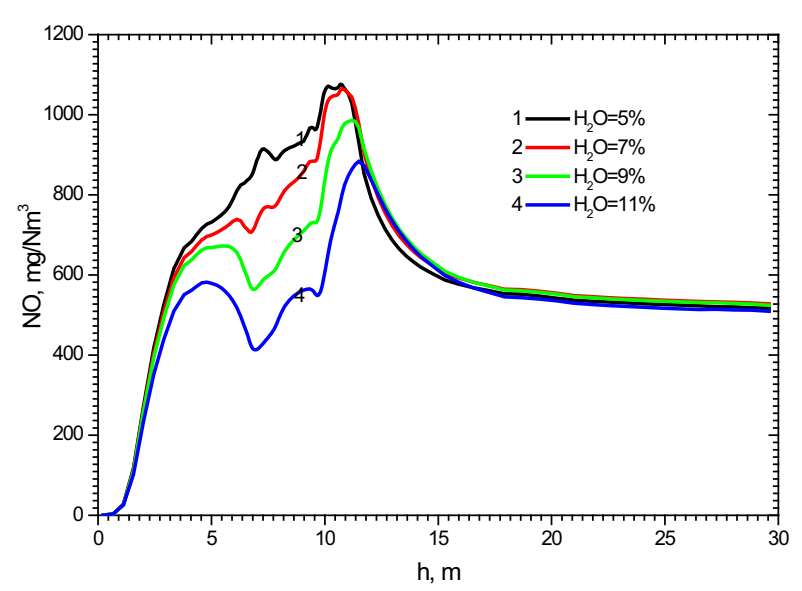

Figure 8. NO concentration distribution along the chamber height for coal burning with different water content.

\section{Conclusions}

In present work CFD simulation tool was used to numerically simulate and analyze low-grade coal combustion and flow properties of industrial furnaces of Kazakhstan Power Plants. The simulations of the furnaces of steam generator of Almaty Power Plant have been carried to predict the influence of different water content in coal on overall furnace operation and formation of combustion products. Results presented in the way of $3 \mathrm{D}$-fields of temperatures, velocities and concentrations of the combustion products $\mathrm{CO}, \mathrm{CO} 2$, $\mathrm{NO}_{\mathrm{x}}$ etc.

CFD prediction of combustion process of combustion chamber can be very useful for engineers to choose an appropriate operating method to successful overall combustion optimization in furnaces to improve energy efficiency and control pollutant emissions.

This work was financially supported by the Ministry of Education and Science of the Republic of Kazakhstan - Grant №0115RK00599.

\section{References}

1. H. Müller, VDI-Verlag. 6, 158 (1992)

2. B.E. Launder, Academic Press, 135 (1972)

3. B. Launder, D. Spalding, J. Comp. Met. Appl. Mech. Eng, (1974)

4. F.G. Lockwood, ASME Paper (1976)

5. J.W. Mitchell, J.M. Tarbell, AIChE J., 28 (1982)

6. R. Leithner, H. Müller, Second M.I.T. Conference on computational Fluid and Solid Mechanics (2003)

7. E.L. Karpenko, V.E. Messerle, et al., 7th International Fall Seminar on Propellants, Explosives and Pyrotechnics, 7 (2007)

8. A.S. Askarova, A.B. Ustimenko, S.A. Bolegenova, V.Yu. Maksimov, J. Thermophysics and aeromechanics, 21 (2014)

9. S.A. Bolegenova, V.Y. Maximov, A. Bekmukhamet, M.T. Beketayeva, Z.K. Gabitova, J. High temperature, 5 (2015) 
10. A.S. Askarova, V.E. Messerle, S.A. Bolegenova, V.Yu. Maximov, Z.Kh. Gabitova, J. High temperature, 53 (2015)

11. V. Maximov, S. Bolegenova, M. Beketayeva, P. Safarik, J. Thermal science, 24 (2015)

12. E.I. Karpenko, V.E. Messerle, J. High Energy Chemistry, 40 (2006)

13. A. Askarowa, M.A. Buchmann, Gesell Energietech, Combustion and incineration - eighteenth dutchgerman conference on flames, VDI Berichte, 1313 (1997)

14. S. Vockrodt, R. Leithner, A. Schiller, VDI Berichte, 1492 (1999)

15. M. Gorokhovski, A. Chtab-Desportes, I. Voloshina, AIP Conference Proceedings, 1207 (2010)

16. V.E. Messerle, A.B. Ustimenko, S.A. Bolegenova, V.Yu. Maximov, J. Thermophysics and Aeromechanics, 23 (2016)

17. S. Bolegenova, M. Beketayeva, Sh. Ospanova, V. Maximov, J. Applied Fluid Mechanics, 9 (2016)

18. M. Beketayeva, S.A. Bolegenova, S. Bolegenova, A. Bekmukhamet, V. Maximov, Int. J. Mech., 7 (2013)

19. Sh. Ospanova, S. Bolegenova, M. Beketayeva, V. Maximov, Int. J. Mech., 8 (2014)

20. S.V. Patankar, Hemisphere Publ. (1980)

21. B.K. Aliarov, B.P. Ustimenko, M.A. Buhman, Report № 01910010550. Kaz. SRIETP (1991) 